\title{
Insects That Feed on Faba Bean in Southern Florida ${ }^{1}$
}

\author{
G. S. Nuessly, M. G. Hentz, R. Beiriger, B. T. Scully, S. E. Halbert, M. C. Thomas, L. A. Stange, G. B. \\ Edwards, J. B. Heppner, G. S. Hodges, and G. J. Steck ${ }^{2}$
}

The faba bean, Vicia faba L., is a cold hardy grain legume originally from central Asia, and now cultivated from tropic to sub-arctic climates (Zeven $\&$ Zhukovsky 1975). This taxa has been artificially divided by seed size into three subspecies (Polhill \& van der Maesen 1985). The broad bean ( $V$. faba var. major Harz) is mostly grown as a grain vegetable because of its large seed size, while the horse bean ( $V$. faba var. equina Pers.) and the pigeon or tick bean ( $V$. faba var. minor Beck) are grown primarily for animal feed or as a green manure crop. In Europe, these two later species are referred to as "field beans" (Bond et al. 1985). With the rare exception of the Austrian pea (Pisum sativum var. arvense (L.) Poiret) used by recreational hunters for deer browse, most legumes grown in Florida are warm season crops and frost intolerant. The faba bean is one of a few freeze tolerant winter legumes that could be integrated into Florida agriculture as either a vegetable or forage crop. It is currently being evaluated as part of three-crop rotational silage cropping system that includes corn (Zea mays L.) and sorghum (Sorghum bicolor Moench) to support the cattle and dairy industries. It has the additional benefits of nitrogen fixation and thus a reasonably low fertility requirement. The information provided below is the result of a two-year study to document insects and insect-transmitted diseases that may have to be considered as pests to this potential crop for Florida (Figure 1). This list of plant and insect feeders will also serve as a reference to help growers, scouts and home gardeners determine which insects they may find on faba beans.

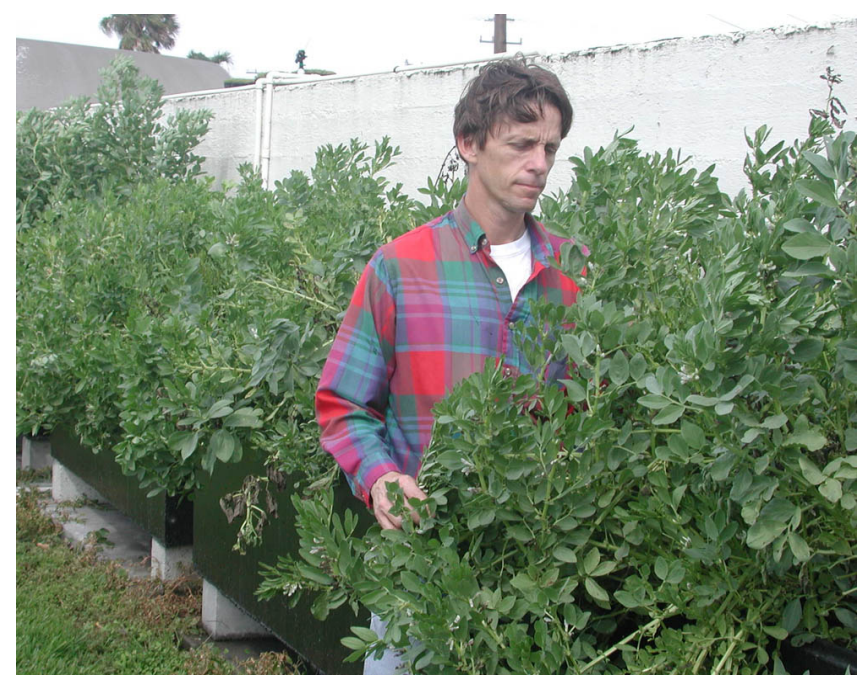

Figure 1. Senior author examining insects and insect-transmitted diseases.

1. This document is ENY-703, one of a series of the Entomology and Nematology Department, Florida Cooperative Extension Service, Institute of Food and Agricultural Sciences, University of Florida. Publication date: August 2005. Please visit the EDIS website at http://edis.ifas.ufl.edu.

2. G. S. Nuessly, Associate Professor, Crop Protection and M.G. Hentz and R. Beiriger, Biologists, Everglades REC, Belle Glade; B.T. Scully, Professor and Center Director, Indian River Research and Education Center, Ft. Pierce; S. E. Halbert, M. C. Thomas (Biological Administrator), L. A. Stange, G. B. Edwards, J. B. Heppner, G. S. Hodges, and G. J. Steck, Division of Plant Industry, Bureau of Entomology, Nematology and Plant Pathology, Florida Department of Agriculture and Consumer Services, Gainesville, Florida 
Fifty-five species of insect herbivores and nectaring predators and parasitoids have been observed feeding on faba bean leaves, stems, flowers, extra-floral nectaries or pods (Table 1) between October and April at the Everglades Research and Education Center, Belle Glade, Florida.

\section{Leaf and Stem Feeders}

Cowpea aphids (Aphis craccivora Koch) (Figure 2) feed mostly on the youngest leaf and stem tissue resulting in stunted terminal growth and distorted leaves. They are known as faba bean pests throughout the Mediterranean and some subtropical and tropical areas where they cause damage from both direct feeding and virus transmission (Cammell \& Way 1983). The pea aphid, Acyrthosiphon pisum, feeds on the underside of leaves in the more protected middle region of the canopy. Pea aphids are known for causing more damage from virus transmission than from direct feeding damage (Cammell \& Way 1983).

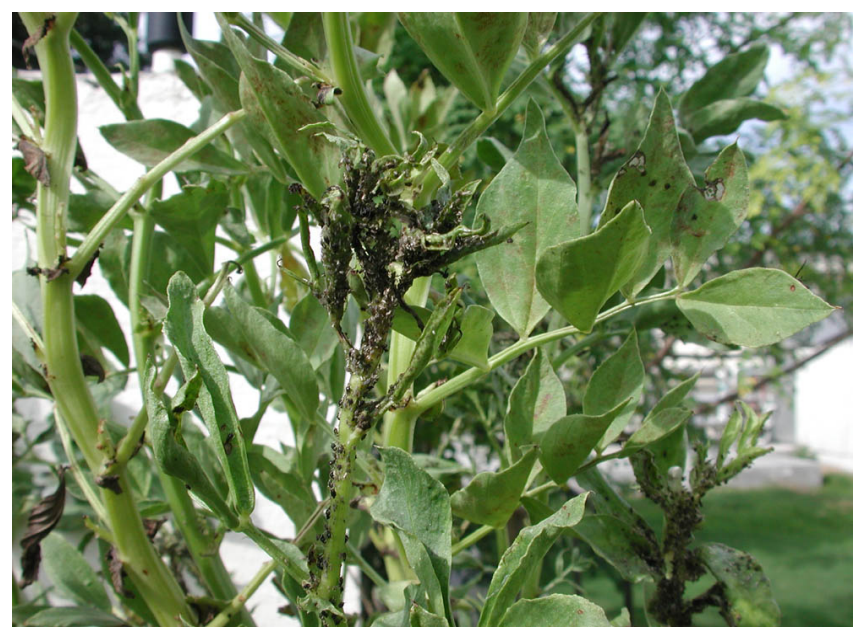

Figure 2. Cowpea aphid.

Bidens mottle mosaic virus (Figure 3 ) is transmitted to faba beans (Baker et al. 2001) by cowpea aphids, pea aphids, green peach aphids (Myzus persicae (Sulzer)) and spirea aphids (Aphis spiraecola Patch). Its causes stunted terminal growth and chlorotic, disfigured leaves and pods.

Virus-infected plants may serve as better hosts of cowpea aphids allowing them to produce more young on infected than on non-infected plants (El-Kady \& Salem 1974).

Two species of leafminers attack leaves, but not pods, of faba bean leaves. Damage includes mines,

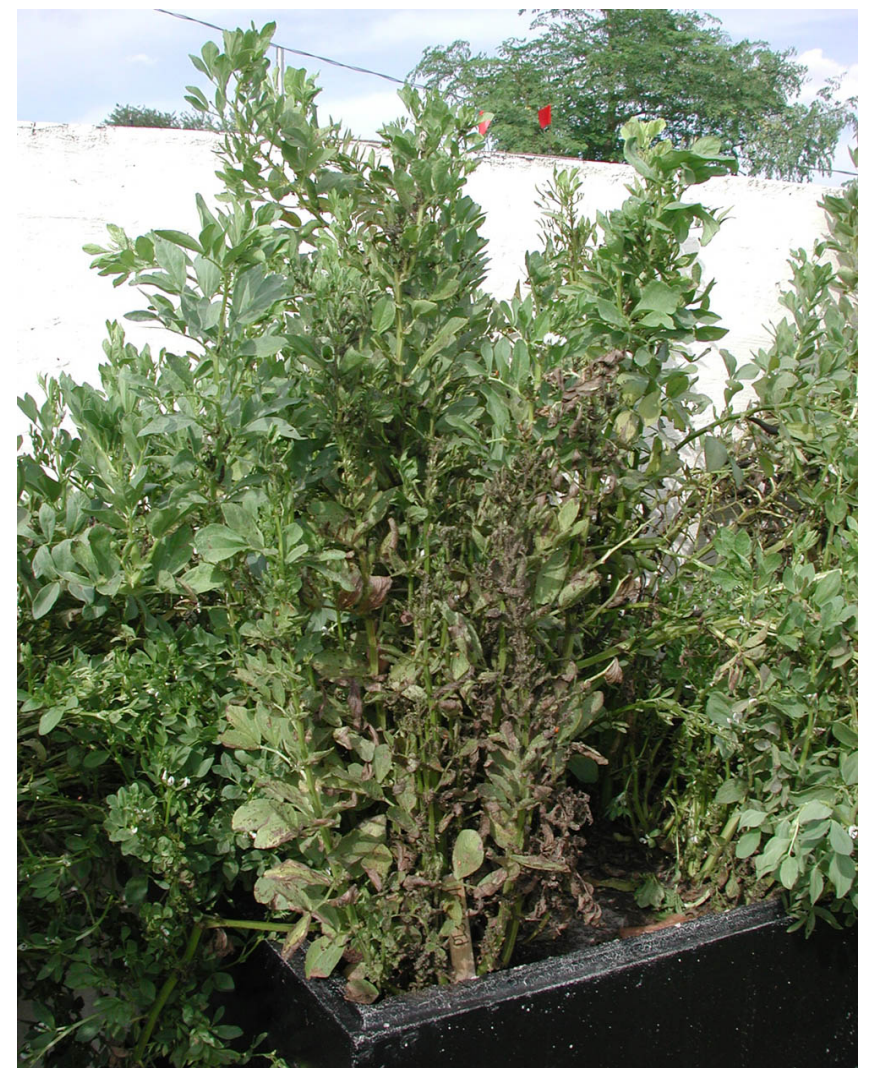

Figure 3. Bidens mottle mosaic virus.

and feeding and egg deposition scars. Mines produced by American serpentine leafminer larvae, Liriomyza trifolii (Burgess), are common during the first months of growth. A second, unidentified species of leafminer fly produced much wider and longer leaf mines lined with a dark residue. These larger mines closely resembled those produced by the blotch leafminer, Amauromyza maculosa (Malloch) (Diptera: Agromyzidae), which occasionally attacks leafy vegetables in our area.

Species from several insect orders chew on faba bean leaves. A grasshopper species, Chortophaga australior Rehn \& Hebard and a katydid, Microcentrum rhombifolium (Saussure), eat large jagged edge sections from leaves. Granulate cutworm larvae, Feltia subterranea (F.), cut off seedling faba beans at their base. The adults of both cucumber beetle species found in southern Florida, banded cucumber beetle (Diabrotica balteata Leconte) and southern corn rootworm $(D$. undecimpunctata howardi Barber), produce irregular sized holes on the edge and middle of the youngest fully expanded leaves. These cucumber beetles have a wide adult host feeding range and D. balteata is a pest of leafy 
vegetables (Nuessly and Webb 2003a) and sweet corn in southern Florida (Nuessly and Webb 2003b). West Indian sugarcane root borers (Diaprepes abbreviatus (L.)) feed on leaf edges. Larvae of the tiger moth (Spilosoma virginica $(\mathrm{F}$.$) ) and Io moth (Automeris io$ io $(\mathrm{F})$.$) have been the only Lepidoptera observed to$ complete development on the plants. Larvae of other species, including the southern armyworm (Spodoptera eridania (Cramer)), were collected on plants, but were likely predated by wasps, beetles, and assassin bugs before they could complete development.

\section{Flower and Nectar Feeders}

Insects found feeding on flowers and nectar include aphids (Figure 4), thrips, beetles, skippers and wasps. The thrips Frankliniella bispinosa (Morgan), F. insularis (Franklin), and F. kelliae (Sakimura) feed on pollen, anthers, and other flower parts, but have not been observed to negatively effect pollination or seed set. Adults of three chafer beetle species feed on pollen and nectar within faba bean flowers. Two of these, Anomala marginata (F.) and Euphoria sepulcralis (F.) (Figure 5), are common flower feeders, and the latter species feeds at ear tips and armyworm feeding holes of sweet corn (Zea mays L.) ears. The third, Trigonopeltastes delta Forster, also feeds on fragrant inflorescences of many plants, including the sable or cabbage palm (Sabal palmetto (Walt. Lodd.)). Adult soldier beetles Chauliognathus marginatus (F.) feed on nectar and pollen within flowers during late afternoon and early evening, when mating pairs are frequently observed. The skipper butterfly Lerema accius (J.E. Smith) nectars from faba bean flowers. Various bees (Anthophoridae, Halictidae and Apidae), paper wasps (Vespidae), spider wasps (Sphecidae), and the cuckoo wasp Chrysis sp. all feed from flowers during the day. Two Chalcidoidae species feed from extra floral nectaries.

\section{Pod Feeders}

Insects that feed on pods composes the largest guild of faba bean herbivores. The stainer Dysdercus mimulus Hussey, four species of leaf-footed bugs and seven species of stink bugs feed on developing pods. Leptoglossus phyllopus (L.) (Figure 6) is the most common and destructive leaf-footed bug. Their

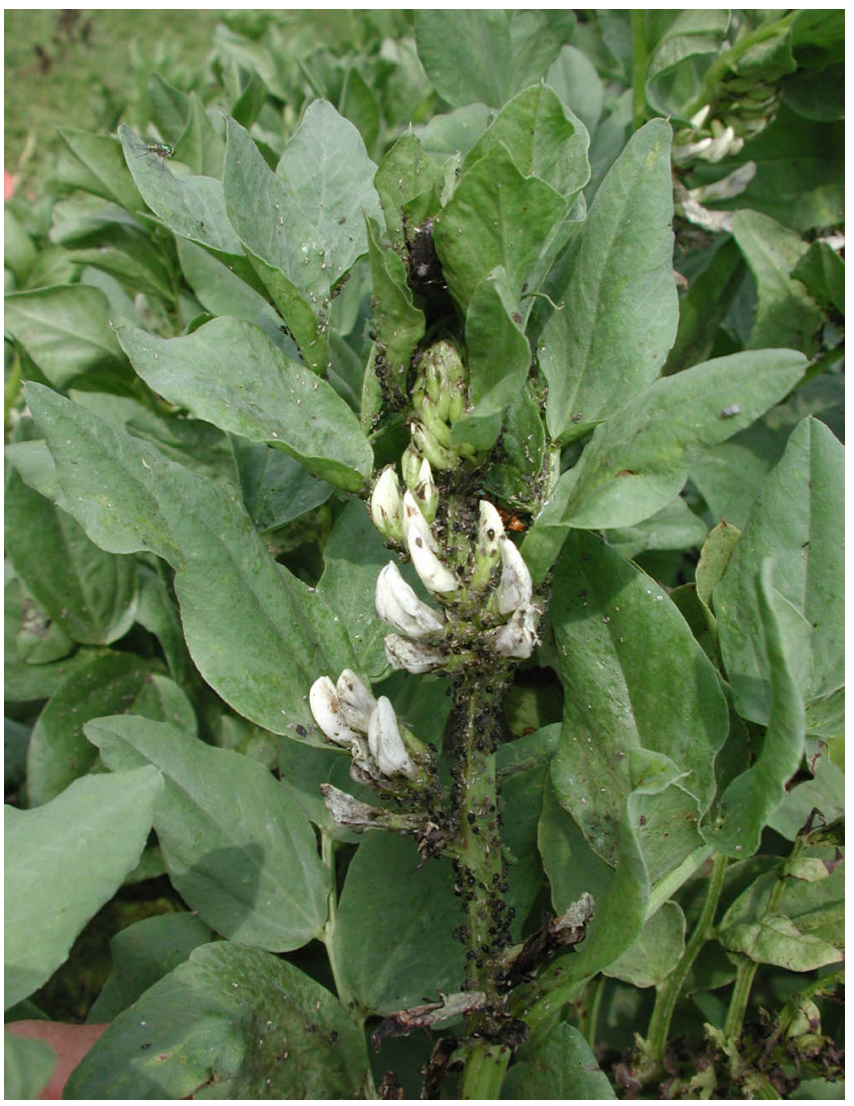

Figure 4. Aphids feeding on flowers and nectar.

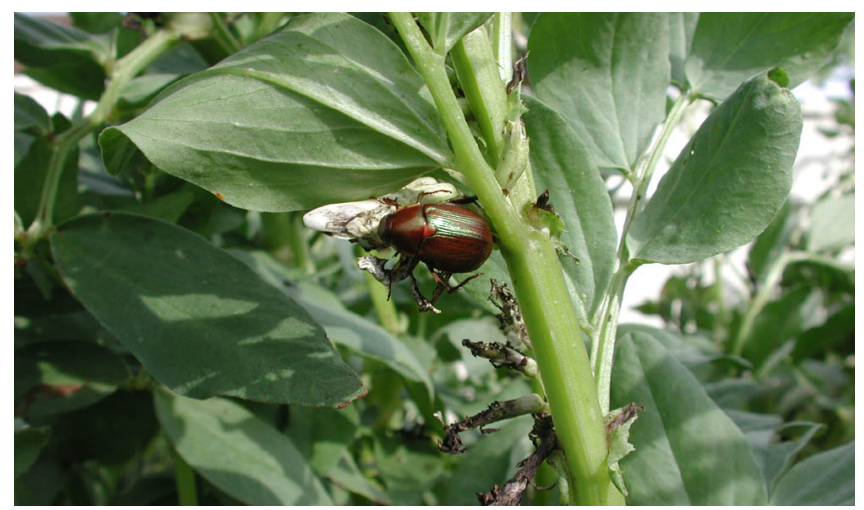

Figure 5. A common flower feeder, Euphoria sepulcralis (F.).

nymphs feed in small groups on pods. Pod feeding by these plant bugs produces raised, pitted black bumps on the pod surface and black spots on developing seeds. This species feeds on a wide variety of cultivated crops, including cowpea (Baranowski \& Slater 1986). The other leaf-footed bugs listed here are more commonly found associated with native plants and are not currently identified as pests of leguminous plants (Baranowski \& Slater 1986). Nymphs and adults of the southern green stink bug (Nezara viridula (L.)) cause pod damage similar to 
that by L. phyllopus. Six other stink bug species feed on pods but have not been observed to reproduce on faba beans. Three of these, Acrosternum hilare (Say) (Simmons \& Yeargan 1990), A. marginatum (Palesot de Bearvois) (Hallman et al. 1985), and Thyanta perditor (F.) (Saunders et al. 1983), are known to cause at least some damage to soybeans or other cultivated beans.

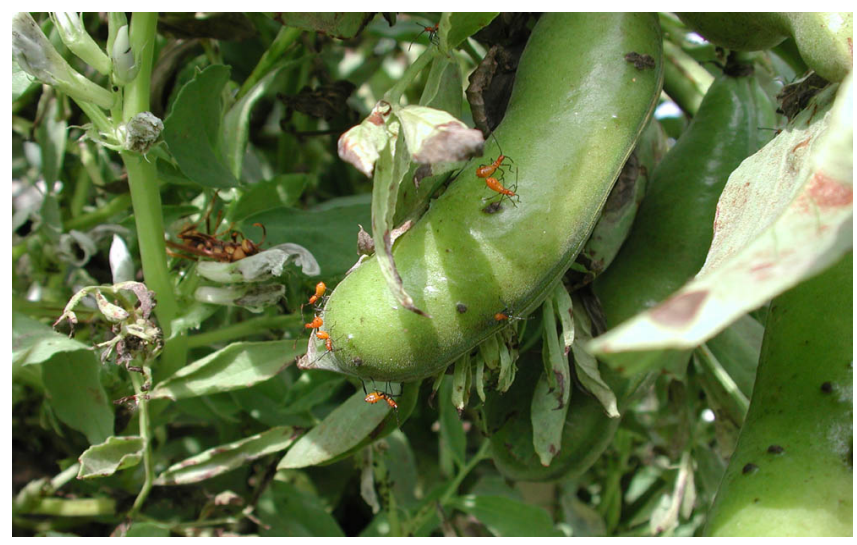

Figure 6. Leptoglossus phyllopus (L.), the most common and destructive leaf-footed bug

\section{Summary}

Several species of insects cause damage to faba bean. Aphid (Aphis craccivora) feeding on terminals and virus infection (Bidens mottle mosaic) by colonizing and non-colonizing aphids reduces the growth and reproduction of the crop. Leaf feeding by cutworms and armyworms can result in reduced stand count and leaf cover if unchecked by natural enemies. Feeding by leaf-footed bugs (Leptoglossus phyllopus) and stink bugs (Nezara viridula) causes damage to seeds developing within pods. Other potential pests to faba bean in Florida include black bean aphids (Aphis fabae Scopoli) and other species with known pest associations with warm season beans in Florida such as melon thrips (Thrips palmi Karney), cowpea curculio weevil (Chalcodermus aeneus Bohemanor), the skipper known as the bean leafroller (Urbanus proteus $(\mathrm{L})$.$) and the snout moth known as the lesser$ cornstalk borer (Elasmopalpus lignosellus (Zeller)) (Capinera 2001). Additional insects may successfully colonize faba beans in other growing areas of Florida.

\section{References Cited}

Baker, C. A., R. N. Raid, and B. T. Scully. 2001. Natural infection of Vicia faba by Bidens mottle virus in Florida. Plant disease. 85 (12): 1290.

Baranowski, R. M., and J. A. Slater. 1986. Coreidae of Florida. (Hemiptera: Heteroptera). Florida Dept. Agric and Consumer Serv., Arthropods of Florida and Neighboring Land Areas, Volume 7, Div. Plant Industry, Gainesville, FL, 82 pp.

Bond, D. A., Lawes, G. C. Hawtin, M. C. Saxena, and J. H. Stephens. 1985. Faba bean (Vicia faba L.), pp. 199-265. In Grain Legume Crops, eds R. J. Summerfield \& E. H. Roberts. Collins, London.

Cammell, M. E., and M. J. Way. 1983. Aphid Pests, pp. 315-346. In Hebblethwaite, P. D. (ed.), The Faba Bean (Vicia faba L.) A Basis for Improvement. Butterworths, London.

Capinera, J. L. 2001. Handbook of Vegetable Pests. Academic Press, San Diego. 729pp.

El-Kady, E. A., and A. A. Salem. 1974. Effect of the presence of broad bean mosaic virus on the biology of Aphis craccivora Koch. (Homoptera: Aphididae). Bull. Soc. Ent. Egypte 57: 319-323.

Hallman, G. J., C. G. Morales, J. M. M. Hollands, and A. Oree. 1985. Infestacion por el chinche verde de frijol Acrosternum marginatum (Palesot de Bearvois) sobre redimientro de Phaseolus vulgaris L.: su efecto. Turrealba 36: 21-24.

Nuessly, G. S., and S. E. Webb. 2003a. Insect Management for Leafy Vegetables (Lettuce, Endive and Escarole). Entomology \& Nematology Department, Florida Cooperative Extension Service, Institute of Food and Agricultural Sciences, University of Florida, ENY-475. http://edis.ifas.ufl.edu/IG161.

Nuessly, G. S., and S. E. Webb. 2003b. Insect Management for Sweet Corn. Entomology \& Nematology Department, Florida Cooperative Extension Service, Institute of Food and Agricultural Sciences, University of Florida, ENY-472. http://edis.ifas.ufl.edu/IG158. 
Polhill, R. M., and L. J. G. van der Maesen. 1985.

Taxonomy of grain legumes, pp. 3-36. In R. J.

Summerfield \& E. H. Roberts (eds,), Grain Legume

Crops. Collins, London.

Saunders, J. L., A. B. S. King, and G. L. Vargus. 1983. Plagas de Cultivos en America Central: Una lista de Referencia. Centro Agronomico Tropical de Investigacion y Ensenanga Boletin Technico, no. 9, Costa Rica, 90 pp.

Simmons, A. M., and K. V. Yeargan. 1990. Effect of combined injuries from defoliation and green stink bug (Hemiptera: Pentatomidae) and influence of field cages on soybean yield and seed quality. J. Econ. Entomol. 83: 599-609.

Zeven, A. C., and P. M. Zhukovsky. 1975.

Dictionary of Cultivated Plants and Their Centers of Diversity Eccluding Ornamentals, Forest Trees, and Lower Plants. Wageningen Cen. Agric. Publ. \& Doc.

Table 1. Insects found feeding on leaves, stems, flowers, extra-floral nectaries and pods of faba beans at Belle Glade, Florida.

\begin{tabular}{|c|c|c|c|c|c|}
\hline Order & Family & Species Name & Common Name & $\begin{array}{l}\text { Life } \\
\text { Stage }\end{array}$ & Plant Part \\
\hline \multirow[t]{2}{*}{ Orthoptera } & Acrididae & $\begin{array}{l}\text { Chortophaga australior } \\
\text { Rehn \& Hebard }\end{array}$ & $\begin{array}{l}\text { short-horned } \\
\text { grasshopper }\end{array}$ & A & Leaf \\
\hline & Tettigoniidae & $\begin{array}{l}\text { Microcentrum rhombifolium } \\
\text { (Saussure) }\end{array}$ & $\begin{array}{l}\text { long-horned } \\
\text { grasshopper }\end{array}$ & $A$ & Leaf \\
\hline \multirow[t]{3}{*}{ Thysanoptera } & \multirow[t]{3}{*}{ Thripidae } & $\begin{array}{l}\text { Frankliniella bispinosa } \\
\text { (Morgan) }\end{array}$ & Florida flower thrips & $L \& A$ & Flower \\
\hline & & $\begin{array}{l}\text { Frankliniella insularis } \\
\text { (Franklin) }\end{array}$ & thrips & A & Flower \\
\hline & & $\begin{array}{l}\text { Frankliniella kelliae } \\
\text { (Sakimura) }\end{array}$ & thrips & A & Flower \\
\hline \multirow[t]{9}{*}{ Hemiptera } & Miridae & Creontiades rubinervis (Stal) & plant bug & A & Leaf \\
\hline & \multirow[t]{2}{*}{ Lygaeidae } & $\begin{array}{l}\text { Oncopeltus cayensis } \\
\text { Torre-Bueno }\end{array}$ & seed bug & A & Stem \& Pod \\
\hline & & Ozophora trinotata Barber & seed bug & A & Leaf \\
\hline & Pyrrhicoridae & Dysdercus mimulus Hussey & stainer & A & Pod \\
\hline & \multirow[t]{4}{*}{ Coreidae } & $\begin{array}{l}\text { Acanthocephala femorata } \\
\text { (F.) }\end{array}$ & leaf-footed bug & $A$ & Pod \\
\hline & & Anasa scorbutica (F.) & leaf-footed bug & A & Pod \\
\hline & & Leptoglossus phyllopus (L.) & leaf-footed bug & $N \& A$ & Pod \\
\hline & & Zicca taeniola (Dallas) & leaf-footed bug & $A$ & Pod \\
\hline & Alydidae & $\begin{array}{l}\text { Stenocoris tipuloides } \\
\text { (DeGeer) }\end{array}$ & broad-headed bug & A & Stem \& Pod \\
\hline
\end{tabular}


Table 1. Insects found feeding on leaves, stems, flowers, extra-floral nectaries and pods of faba beans at Belle Glade, Florida.

\begin{tabular}{|c|c|c|c|c|c|}
\hline Order & Family & Species Name & Common Name & $\begin{array}{l}\text { Life } \\
\text { Stage }\end{array}$ & Plant Part \\
\hline & \multirow[t]{7}{*}{ Pentatomidae } & Acrosternum hilare (Say) & green stink bug & $N \& A$ & Pod \\
\hline & & $\begin{array}{l}\text { Acrosternum marginatum } \\
\text { (Palesot de Bearvois) }\end{array}$ & stink bug & $A$ & Pod \\
\hline & & Edessa bifida (Say) & stink bug & A & Pod \\
\hline & & Euschistus ictericus (L.) & stink bug & $A$ & Pod \\
\hline & & $\begin{array}{l}\text { Euschistus quatrator } \\
\text { Raulston }\end{array}$ & stink bug & $A$ & Pod \\
\hline & & Nezara viridula (L.) & $\begin{array}{l}\text { Southern green } \\
\text { stink bug }\end{array}$ & $N \& A$ & Pod \\
\hline & & Thyanta perditor (F.) & stink bug & A & Pod \\
\hline & \multirow[t]{2}{*}{ Cicadellidae } & $\begin{array}{l}\text { Draeculocephala mollipes } \\
\text { (Say) }\end{array}$ & leafhopper & $N \& A$ & Leaf \\
\hline & & Gypona sp. & leafhopper & $N \& A$ & Leaf \\
\hline & \multirow[t]{2}{*}{ Aphidae } & $\begin{array}{l}\text { Acyrthosiphon pisum } \\
\text { (Harris) }\end{array}$ & pea aphid & $N \& A$ & Leaf \\
\hline & & Aphis craccivora Koch & cowpea aphid & $N \& A$ & Leaf \& Stem \\
\hline & Pseudococcidae & Planococcus citri (Risso) & mealybug & $N \& A$ & Pollen/Nectar \\
\hline \multirow[t]{7}{*}{ Coleoptera } & \multirow[t]{3}{*}{ Scarabaeidae } & Anomala marginata (F.) & shining leaf chafer & $A$ & Pollen/Nectar \\
\hline & & Euphoria sepulcralis (F.) & flower chafer & $A$ & Pollen/Nectar \\
\hline & & $\begin{array}{l}\text { Trigonopeltastes delta } \\
\text { Forster }\end{array}$ & flower chafer & $A$ & Pollen/Nectar \\
\hline & Cantharidae & $\begin{array}{l}\text { Chauliognathus marginatus } \\
\text { (F.) }\end{array}$ & soldier beetle & A & Pollen/Nectar \\
\hline & \multirow[t]{2}{*}{ Chrysomelidae } & Diabrotica balteata Leconte & $\begin{array}{l}\text { banded cucumber } \\
\text { beetle }\end{array}$ & A & Leaf \\
\hline & & $\begin{array}{l}\text { Diabrotica undecimpunctata } \\
\text { howardi Barber }\end{array}$ & $\begin{array}{l}\text { southern corn } \\
\text { rootworm }\end{array}$ & $A$ & Leaf \\
\hline & Curculionidae & Diaprepes abbreviatus (L.) & sugarcane root borer & $A$ & Leaf \\
\hline \multirow[t]{5}{*}{ Lepidoptera } & Arctiidae & Spilosoma virginica (F.) & tiger moth & $\mathrm{L}$ & Leaf \\
\hline & \multirow[t]{2}{*}{ Noctuidae } & Feltia subterranea (F.) & granulate cutworm & $\mathrm{L}$ & Seedling stem \\
\hline & & $\begin{array}{l}\text { Spodoptera eridania } \\
\text { (Cramer) }\end{array}$ & southern armyworm & $\mathrm{L}$ & Leaf \\
\hline & Saturniidae & Automeris io io (F.) & lo moth & $\mathrm{L}$ & Leaf \\
\hline & Hesperiidae & Lerema accius (J. E. Smith) & skipper & $A$ & Flower \\
\hline \multirow[t]{3}{*}{ Diptera } & Otitidae & Euxesta annonae (F.) & picture-winged fly & A & $\begin{array}{l}\text { Extra floral } \\
\text { nectary }\end{array}$ \\
\hline & Tephritidae & Xanthaciura insecta (Loew) & fruit fly & A & $\begin{array}{l}\text { Extra floral } \\
\text { nectary }\end{array}$ \\
\hline & Agromyzidae & Liriomyza trifolii (Burgess) & serpentine leafminer & $L \& A$ & Leaf \\
\hline \multirow[t]{2}{*}{ Hymenoptera } & \multirow[t]{2}{*}{ Chalcididae } & Brachymeria sp. & $\begin{array}{l}\text { chalcidid parasitic } \\
\text { wasp }\end{array}$ & $\mathrm{A}$ & $\begin{array}{l}\text { Extra floral } \\
\text { nectary }\end{array}$ \\
\hline & & Conura sp. & $\begin{array}{l}\text { chalcidid parasitic } \\
\text { wasp }\end{array}$ & A & $\begin{array}{l}\text { Extra floral } \\
\text { nectary }\end{array}$ \\
\hline
\end{tabular}


Table 1. Insects found feeding on leaves, stems, flowers, extra-floral nectaries and pods of faba beans at Belle Glade, Florida.

\begin{tabular}{|c|c|c|c|c|c|}
\hline Order & Family & Species Name & Common Name & $\begin{array}{l}\text { Life } \\
\text { Stage }\end{array}$ & Plant Part \\
\hline & Chrysidae & Chrysis sp. & cuckoo wasp & A & Nectar \\
\hline & \multirow[t]{2}{*}{ Halictidae } & $\begin{array}{l}\text { Agapostemon splendens } \\
\text { (Lepeletier) }\end{array}$ & solitary bee & A & Nectar \\
\hline & & Halictus sp. & solitary bee & A & Nectar \\
\hline & Anthophoridae & Xylocopa micans Lepeletier & carpenter bee & 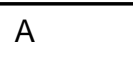 & Pollen/nectar \\
\hline & Apidae & Apis mellifera L. & honey bee & $\mathrm{A}$ & Pollen/nectar \\
\hline & \multirow[t]{5}{*}{ Vespidae } & Eumenes fraternus Say & paper wasp & A & Nectar \\
\hline & & $\begin{array}{l}\text { Pachyodynerus nasidens } \\
\text { (Latreille) }\end{array}$ & paper wasp & A & Nectar \\
\hline & & Polistes dorsalis (F.) & paper wasp & A & Nectar \\
\hline & & Polistes major Beauvois & paper wasp & A & Nectar \\
\hline & & Polistes metricus Say & paper wasp & A & Nectar \\
\hline & Sphecidae & Liris sp. & spider wasp & A & Nectar \\
\hline
\end{tabular}

